\title{
ESTUDIO DE ESTABILIDAD DE VEHÍCULOS PESADOS
}

\author{
STABILITY STUDY OF HEAVY VEHICLES
}

\section{PhD. Gonzalo G Moreno C*, PhD. Elkin G Flórez* PhD. César A Peña **}

* Universidad de Pamplona, Facultad de Ingenierías y Arquitectura, Programa de Ingeniería Mecánica, Grupo de Investigación en Ingeniería Mecánica de la Universidad de Pamplona (GIMUP). Pamplona - Colombia, Km 1 vía Bucaramanga. Tel.: (+57 7) 568 5303, Fax: (+57 7) 568 5303, Ext. 144

E-mail: gmoren,eflorez@unipamplona.edu.co

** Universidad de Pamplona, Facultad de Ingenierías y Arquitectura, Programa de Ingeniería Mecatrónica, Pamplona - Colombia, Km 1 vía Bucaramanga. Tel.: (+57 7) 568 5303, Fax: (+57 7) 568 5303, Ext. 144.

E-mail: cesarapc@unipamplona.edu.co

Resumen: La estabilidad de vehículos pesados es un tema ampliamente estudiado en la actualidad, dado que de esta forma es posible comprender una de las principales causas de accidentes vehiculares: el vuelco de vehículos. El objetivo principal de los investigadores es determinar una forma de predecir el comportamiento de un vehículo bajo ciertas condiciones de operación. En este sentido, en este artículo se compara el análisis clásico de estabilidad de un vehículo en dos dimensiones y un análisis de estabilidad de un modelo tridimensional utilizando la misma metodología.

Palabras clave: Vehículos pesados, estabilidad, límite de vuelco estático, límites de velocidad, seguridad vial.

\begin{abstract}
The heavy vehicle stability is a widely studied topic today, since it is responsible for a better understanding as it is one of the main vehicular accident rate problems in the world: the rollover accident. The main goal for researchers is to determine a way to predict vehicle behavior under some circumstances. In this sense, this paper compares the classic vehicle stability analysis of a model in two dimensions, and the vehicle stability analysis of a three-dimensional model using the same methodology.
\end{abstract}

Keywords: Heavy vehicles, stability, static rollover threshold, speed limits, road safety.

\section{INTRODUCCIÓN}

La estabilidad de vehículos pesados es un tema de gran interés en la actualidad, ya que en ciertas trayectorias del movimiento de un vehículo, como lo son las curvas o las maniobras evasivas y la fuerza inercial que actúa en el centro de gravedad del vehículo (CG) hacen que se presenten dos tipos de accidentes: el vuelco o la salida de la carretera

Estos dos tipos de accidentes, aunque no son tan comunes, por su gravedad son los que producen un 
mayor número de muertos, heridos y perdidas económicas en las vías, con respecto a otros accidentes como lo son los choques, atropellos e incendios de vehículos (IPEA, 2015; CAN, 2013; Garbarino, 2016).

Teniendo en cuenta el primero de esta clase de accidentes, el vuelco de los vehículos pesados se puede clasificar en dos categorías: vuelco imprevisible y vuelco previsible.

El vuelco imprevisible se presenta cuando un vehículo realiza maniobras evasivas (como esquivar personas o imperfecciones de las vías o realizar maniobras de zig-zag), estas maniobras son difíciles de estudiar, ya que la fuerza inercial depende de muchas variables asociadas con el tipo de maniobra.

Por otro lado, el vuelco previsible se presenta únicamente cuando los vehículos realizan una trayectoria curva, en este caso la fuerza inercial aplicada sobre el centro de gravedad del vehículo (CG) depende del radio de la curva y de la velocidad del vehículo, estas variables son controlables y permiten ser estudiadas con el fin de establecer límites de velocidad que hagan más seguro el transito por las vías actuales.

En este sentido, una de las principales metodologías para analizar la tendencia al vuelco de los vehículos pesados es el estudio de estabilidad lateral, para lo cual se utiliza el factor denominado Static Rollover Thereshold (SRT) por su nombre en inglés o límite de vuelco estático, este factor es altamente dependiente de la aceleración centrípeta $\left(a_{y}\right)$ expresada en función de la gravedad $(g)$ y de la ubicación del centro de gravedad del vehículo (CG), el cual a su vez depende de otros factores como el tipo de carga, la distribución de la carga, de la suspensión, los neumáticos, etc.,(Winkler, 1987; Gillespie, 1992; Hac, 2002).

Debido a las dimensiones de los vehículos, a la complejidad de estos y a los costos que puedan acarrear el realizar pruebas dinámicas de vehículos pesados, la gran mayoría de los estudios desarrollados se basan en modelos matemáticos que permiten simular muchas de las variables que intervienen en este tipo de accidente, permitiendo de esta forma entender mejor lo que ocurre cuando esta situación se presenta.

En este sentido, varios de los modelos de estabilidad encontrados en la literatura, son modelos bidimensionales (2D) (Gillespie, 1992; Winkler, 2000; Chang, 2001; Rill, 2011; Moreno et al., 2016), los cuales permiten determinar como algunas de las características del vehículo y de la carretera, tales como la suspensión, los neumáticos y el ángulo de declive lateral de la carretera o peralte, influencian en el cálculo del factor de estabilidad. Sin embargo, hay que tener en cuenta que estos estudios asumen que los vehículos tienen únicamente dos puntos de contacto con la carretera, lo cual es cuestionable, ya que todos los vehículos automotores por lo general tienen cuatro puntos de contacto con la carretera, adicionalmente si se tiene en cuenta la flexibilidad del chasis del vehículo y la ubicación longitudinal del centro de gravedad (CG), estas características pueden ser determinantes al momento de calcular el factor de estabilidad (Moreno et al., 2016).

Teniendo en cuenta todo lo expuesto anteriormente, en el presente artículo se hace un análisis y comparación de dos modelos rígidos de estabilidad vehicular, y se demuestra como el modelo de estabilidad tridimensional (3D) permite obtener mejores resultados del factor de estabilidad que el modelo bidimensional (2D).

El artículo está dividido en las siguientes secciones: la Sección 2 muestra en detalle los modelos planteados y el cálculo del factor de estabilidad para cada uno de ellos y la comparación de estos, en la Sección 3 se muestra un caso de estudio de los modelos desarrollados, y por último en la Sección 4 se presentan las conclusiones del trabajo.

\section{MODELOS DE ESTABILIDAD VEHICULAR}

Para la determinación del factor de estabilidad de los modelos bidimensional y tridimensional se utilizara un vehículo rígido, y se empleara la forma clásica (Modelo Newtoniano) para la determinación del factor. A continuación se detalla cada uno de los modelos propuestos.

\subsection{Modelo bidimensional}

Para la determinación del factor de estabilidad (SRT), se supone un vehículo completamente rígido realizando una curva hacía la izquierda, el cual tiene dos puntos de apoyo con la carretera $\left(F_{z 1}\right.$ y $F_{z 2}$ ) y sobre el cual actúan únicamente la fuerza inercial $\left(m a_{y}\right)$ y el peso del vehículo $(W=m g)$, como se indica en la Fig. 1. 


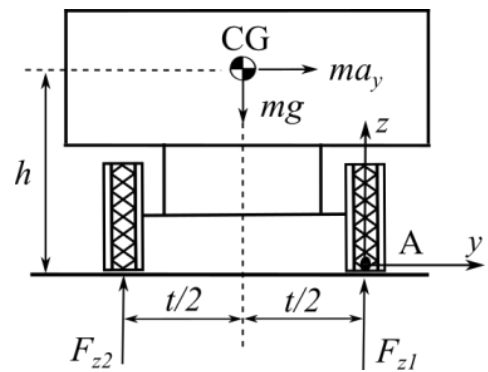

Fig. 1. Modelo de estabilidad bidimensional.

Durante la maniobra descrita, las fuerzas de fricción presentes en la interacción entre los neumáticos y la carretera, hacen que el vehículo se balancee hacia la derecha, ocasionando muchas veces que el vehículo gire entorno de la rueda que se encuentra en la parte externa de la curva (Gillespie, 1992); teniendo en cuenta este fenómeno y considerando la ecuación de momentos entorno del punto A (contacto de la rueda con la carretera en la parte externa de la curva) se tiene:

$$
\sum M_{A}=m g\left(\frac{t}{2}\right)-m a_{y}(h)-F_{z 2}(t)=0
$$

donde $t$ es la distancia lateral existente entre los puntos de contacto de los neumáticos de un mismo eje, $h$ es la altura del centro de gravedad (CG) con respecto al punto de referencia $A$ y $F_{z 2}$ es la fuerza de reacción o carga que soporta el neumático de la parte interna de la curva.

Considerando que inicialmente el peso del vehículo $(W)$ (Fig. 1) es distribuido uniformemente en ambos lados de esté, la fuerza inicial $\left(F_{z i}^{\text {start }}\right)$ que soportan cada uno de los neumáticos puede ser definida como:

$$
F_{z 1}^{\text {start }}=F_{z 2}^{\text {start }}=\frac{1}{2} m g
$$

Cuando el vehículo realiza la curva, la carga que soporta la rueda externa de la curva $\left(F_{z I^{\text {start }}}\right)$ es incrementada gradualmente por la tasa de transferencia de carga $\left(\Delta F_{z}\right)$; y la carga que soporta la rueda interna de la curva $\left(F_{z 2}{ }^{\text {start }}\right)$ se disminuye en la misma proporción (Lui et al., 1997; Kamnik et al., 2003; Rill, 2011; Imine et al., 2014), como es mostrado en la Ecuación 3.

$$
F_{z 1}=F_{z 1}^{\text {stant }}+\Delta F_{z} \wedge F_{z 2}=F_{z 2}^{\text {stant }}-\Delta F_{z}
$$

En el límite de vuelco del vehículo toda la carga se transfiere de un lado al otro del vehículo, lo cual se conoce como coeficiente de transferencia lateral de carga o Lateral Load Transfer ( $L L T)$ por su nombre en inglés, por consiguiente se tiene:

$$
\Delta F_{z}=\frac{1}{2} m g
$$

De este modo el coeficiente de transferencia lateral de carga $(L L T)$ se define como la proporción de carga que transfiere de un lado al otro del vehículo, y se representa por la siguiente ecuación:

$$
L L T=\frac{F_{z 1}-F_{z 2}}{F_{z 1}+F_{z 2}}
$$

Teniendo en cuenta que en el límite de vuelco del vehículo toda la carga se transfiere de un lado al otro del vehículo, lo cual hace que la carga $F_{z 2}$ tiende a cero, por consiguiente la transferencia de carga es completa $(L L T=1)$; así, aplicando esta condición en la Ecuación 1, el factor de estabilidad bidimensional se puede expresar como:

$$
S R T_{2 D}=\frac{a_{y}}{g}=\frac{t / 2}{h}
$$

En la Ecuación 6 se puede observar que el factor de estabilidad (SRT) depende de la aceleración centrípeta $\left(a_{y}\right)$ y de la ubicación del centro de gravedad del vehículo.

\subsection{Modelo tridimensional}

Utilizando la misma metodología descrita para el modelo bidimensional, y conforme a los parámetros indicados en la Fig. 2; considerando el balance de fuerzas (momentos) entorno del eje longitudinal que pasa por los puntos de contacto con la carretera con los neumáticos que se encuentran en la parte externa de la curva (1 y 4$)$, se tiene:

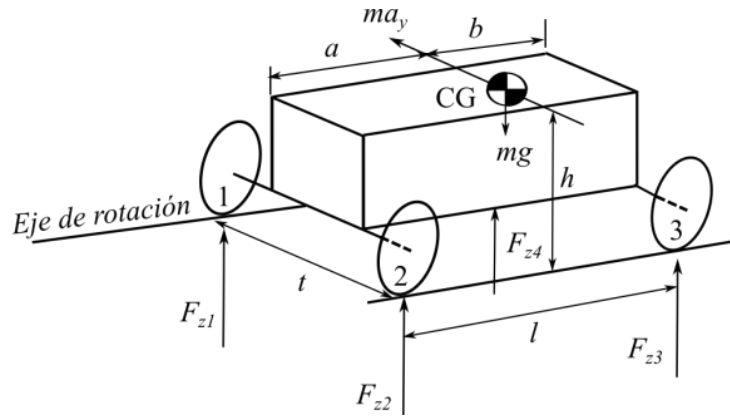

Fig. 2. Modelo de estabilidad tridimensional. 


$$
\begin{gathered}
\sum M_{E J E}=m g\left(\frac{t}{2}\right)-m a_{y}(h) \\
-F_{z 2}(t)-F_{z 3}(t)=0
\end{gathered}
$$

Considerando que inicialmente el peso del vehículo $(W)$ es distribuido uniformemente en la parte trasera y delantera del vehículo, la fuerza inicial $\left(F_{z i}{ }^{\text {start }}\right)$ que soportan cada uno de los neumáticos puede ser definida como:

$$
\begin{aligned}
& F_{z 1}^{\text {stant }}=F_{z 2}^{\text {start }}=\frac{b}{2 l} m g \\
& F_{z 3}^{\text {start }}=F_{z 4}^{\text {start }}=\frac{a}{2 l} m g
\end{aligned}
$$

Considerando la flexibilidad del chasis y de acuerdo a las investigaciones realizadas por Kamnik et al., 2003 y Rill, 2011, las cuales indican que la transferencia de carga (LLT) en la parte trasera del vehículo es mayor porcentualmente que la ocurrida en la parte delantera; para una transferencia completa en la parte trasera del vehículo $\left(L L T_{\text {rear }}=1, F_{z 3}=0\right)$ y para una transferencia incompleta en la parte delantera del vehículo $\left(L L T_{\text {front }}=x, F_{z 2}=(1-x) F_{z 2}{ }^{\text {start }}\right)$; de las Ecuaciones 7 y 8 se tiene:

$$
m g\left(\frac{t}{2}\right) \quad m a_{y}(h) \quad(1 \quad x) \frac{a}{2 l} m g(t)=0
$$

Donde $a$ es la distancia longitudinal entre el eje delantero y el centro de gravedad del vehículo (CG) y $l$ es la distancia longitudinal entre los ejes del vehículo; reorganizando la Ecuación 8, el factor de estabilidad tridimensional $\left(S R T_{3 D}\right)$ está dado por la siguiente ecuación:

$$
S R T_{3 D}=\frac{a_{y}}{g}=\frac{t / 2}{h}\left(1-\frac{(1-x) a}{l}\right)
$$

Analizando y comparando las Ecuaciones 6 y 9 se puede observar que el factor de estabilidad tridimensional $\left(S R T_{3 D}\right)$ es menor que el factor de estabilidad bidimensional $\left(S R T_{2 D}\right)$, esto indica que el vuelco de un vehículo es un fenómeno tridimensional y que se debe tener en cuenta la ubicación longitudinal del centro de gravedad (CG) y la transferencia de carga delantera en el vehículo.

Es importante también resaltar que el factor de estabilidad tridimensional permite entender mucho mejor esta clase de accidentes y brindar mayor seguridad vial cuando se transita por una vía (Duarte et al., 2015; Muñoz et al., 2012). Teniendo en cuenta que la aceleración centrípeta es función de la velocidad del vehículo ( $v$ ) y del radio de la curva ( $r$ ) (Castro et. al., 2013), se puede determinar la velocidad límite con la que un vehículo puede realizar una curva de radio $r$ (Torres et al., 2013; Correa et al., 2010), de la Ecuación 9 se tiene:

$$
\begin{gathered}
S R T_{3 D}=\frac{a_{y}}{g}=\frac{v^{2} / r}{g} \\
v_{\text {linite }}=\sqrt{g \times S R T_{3 D} \times r}
\end{gathered}
$$

\begin{tabular}{|c|c|c|}
\hline Parámetro & Cantidad & Unidades \\
\hline Peso $(W)$ & 260563.41 & $\mathrm{~N}$ \\
\hline Altura del CG $(h)$ & 2.74 & $\mathrm{~m}$ \\
\hline $\begin{array}{c}\text { Distancia lateral entre } \\
\text { ruedas }(t)\end{array}$ & 1.83 & $\mathrm{~m}$ \\
\hline $\begin{array}{c}\text { Distancia longitudinal } \\
\text { CG }(a)\end{array}$ & 7 & $\mathrm{~m}$ \\
\hline $\begin{array}{c}\text { Distancia longitudinal } \\
\text { ejes }(l)\end{array}$ & 13 & $\mathrm{~m}$ \\
\hline Fuerzas $\boldsymbol{F}_{z 1}{ }^{\text {start }}, \boldsymbol{F}_{z 2^{\text {start }}}$ & 60130.02 & $\mathrm{~N}$ \\
\hline Fuerzas $\boldsymbol{F}_{z 3^{\text {start }}}, \boldsymbol{F}_{z 4^{\text {start }}}$ & 70151.69 & $\mathrm{~N}$ \\
\hline
\end{tabular}

\section{CASO DE ESTUDIO}

Para un mejor entendimiento de las ecuaciones de estabilidad (Ecuaciones 6 y 9), se presenta un vehículo completamente rígido (Fig. 2) con los parámetros indicados en la Tabla 1.

De acuerdo con el estudio desarrollado por (Kamnik et al., 2003), cuando un vehículo está realizando una maniobra circular, en estado estable con velocidad constante (Steady State Circular Tests, ISO-14792, 2011) se estima que la transferencia de carga en el eje trasero del vehículo es completa $\left(L L T_{\text {rear }}=1, F_{z 3}=0\right)$ y la transferencia de carga en el eje delantero es del $70 \%$, por consiguiente $L L T_{\text {front }}=0.7$ y $F_{z 2}=(1-x) F_{z 2}$ start .

Teniendo en cuenta los parámetros de la Tabla 1 y la transferencia de carga recomendada para el eje delantero del vehículo $\left(L L T_{\text {front }}=0.7\right)$, la Tabla 2 muestra los resultados obtenidos del factor de estabilidad para los modelos bidimensional y tridimensional, Ecuaciones 6 y 9 respectivamente.

Tabla 2: Factores de estabilidad (SRT) 


\begin{tabular}{ccc}
\hline Factor de estabilidad & $\boldsymbol{S R T}_{2 D}$ & $\boldsymbol{S R T}_{3 D}$ \\
\hline Modelo & 0.334 & 0.28 \\
\hline
\end{tabular}

La Tabla 2 muestra que el factor de estabilidad tridimensional $\left(S R T_{3 D}\right)$ se disminuye cerca del $16 \%$ con respecto al factor de estabilidad bidimensional $\left(S R T_{2 D}\right)$, adicionalmente en la Fig. 3 se pueden observar los límites de velocidad para un vehículo realizando curvas de diferentes radios empleando los factores de estabilidad 2D y 3D.

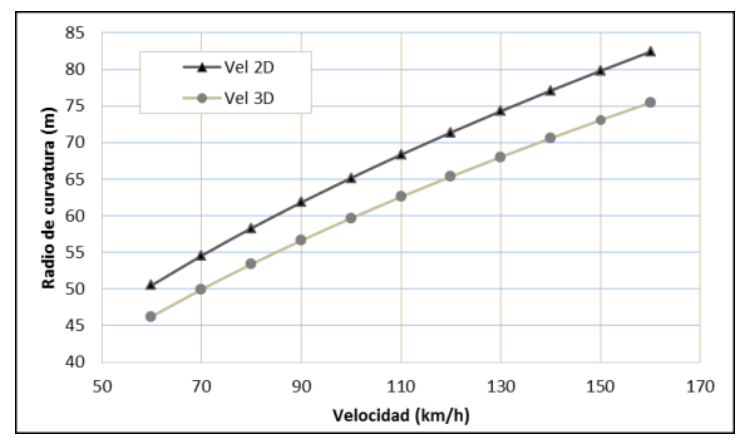

Fig. 3. Limites de velocidad para diferentes radios de curvatura.

Realizando un análisis estadístico de los datos obtenidos y graficados en la Fig. 3 se puede determinar que utilizando el factor de estabilidad tridimensional $\left(S R T_{3 D}\right)$, los límites de velocidad se reducen en promedio $6 \mathrm{~km} / \mathrm{h}$ con respecto a los calculados con el mismo radio de curvatura y con el factor de estabilidad bidimensional $\left(S R T_{2 D}\right)$.

Este análisis permite comprender mejor el fenómeno de vuelco de los vehículos pesados, al mismo tiempo que garantiza el aumento de la seguridad vial ya que disminuye los límites de velocidad con el cual los vehículos pueden realizar una curva.

Es importante recalcar que el vehículo utilizado es un modelo rígido, y que para obtener resultados más próximos a la realidad del fenómeno se deben tener en cuenta la influencia de la suspensión, los neumáticos y del tipo de carga en el cálculo del factor de estabilidad del vehículo. En este sentido se deben utilizar métodos de análisis más avanzados, como el Método de Davies, el cual es presentado por Moreno et al. (2017) en su estudio de estabilidad.

\section{CONCLUSIONES}

En el presente artículo se pudo detallar como a través de los análisis de estabilidad vehicular tridimensionales se puede comprender mejor el vuelco de los vehículos pesados, garantizando de esta forma el aumento en la seguridad vial.

A través del análisis de estabilidad tridimensional se pudo conocer que la ubicación longitudinal del centro de gravedad del vehículo, la flexibilidad del chasis y la transferencia de carga en la parte delantera del vehículo juegan un papel importante al momento de calcular dicho factor.

El cálculo correcto de los límites de velocidad garantizara la movilidad segura de los vehículos por las vías, disminuyendo de esta forma los índices de accidentalidad vial.

\section{REFERENCIAS}

CAN (2013). Accidentes de Tránsito en la Comunidad Andina - Documento Estadístico

Castro, R.; Moreno, J.; Gonzalez, H.; Lengerke, O. (2013). Fuzzy speed control for trajectory follow-up of a robot moving on vertical surfaces. Revista Colombiana de Tecnologias de Avanzada. ISSN: 1692-7257 - v. 2, - n. 22, p 34-41.

Chang, T. (2001). Effect of Vehicles' Suspension on Highway Horizontal Curve Design. Journal of Transportation Engineering, v. 127, n. 1, p. 89-91.

Correa, D.; Sanabria, F.; Sandoval, W.; Claros, R. (2010). Cooperative service robots. Revista Colombiana de Tecnologias de Avanzada. ISSN: 1692-7257 - v. 2, - n. 16, p 119-127.

Duarte, T.; Mendoza, Y.; Arévalo, A.; Guerrero, T. (2015). Modelation of Modal Election for Urban Public Transport Based on the Introduction of a New Mode. Revista Colombiana de Tecnologias de Avanzada. ISSN: 1692-7257 - v. 1, - n. 25, p 83-91.

Garbarino, S.; Durando, P.; Guglielmi, O.; Dini, G.; Bersi, F.; Fornarino, S.; Toletone, A.; Chiorri, C.; Magnavita, N. (2016) Sleep Apnea, Sleep Debt and Daytime Sleepiness Are Independently Associated with Road Accidents. A Cross-Sectional Study on Truck Drivers. PLOS ONE, v 11, n 11 , p. 1-12, doi:10.1371/journal. pone. 0166262

Gillespie, T. D. (1992) Fundamentals of Vehicle Dynamics. 7th. ed. Warrendale, PA.: SAE International, 1992. ISBN 1560911999

Hac, A. (2002) Rollover Stability Index Including Effects of Suspension Design. SAE 
International - SAE 2002 World Congress, Detroit-USA, March 4-7.

Imine, H.; Benallegue, A.; Madani, T.; Srairi, S. (2014). Rollover risk prediction of heavy vehicle using high order sliding mode observer: experimental results. IEEE Transactions on Vehicular Technology, v. 63, n. 6, p. 2533-2543.

IPEA. (2015). Estimativa dos Custos dos Acidentes de Trânsito no Brasil com Base na Atualização Simplificada das Pesquisas Anteriores do Ipea. IPEA - Instituto de Pesquisa Econômica Aplicada. Brasília.

ISO-14792. (2011). Heavy commercial vehicles and buses - Steady state circular tests. International Organization for Starndardization. Geneva, Switzerland.

Kamnik, R.; Boettiger, F.; Hunt, K. (2003). Roll dynamics and lateral load transfer estimation in articulated heavy freight vehicles. Proceedings of the Institution of Mechanical Engineers, Part D: Journal of Automobile Engineering, Sage Publications Sage UK: London, England, v. 217, n. 11, p. 985-997.

Lui, P.; Rakheja, S.; Ahmed, A. (1997). Detection of Dynamic Roll Instability of Heavy Vehicles for Open-Loop Rollover Control. In: 1997 International Truck and Bus Meeting. Cleveland, Ohio.: SAE Special Publications 1308 - SAE Paper No. 973263, 1997. p. 105112.

Moreno, G. G., Barreto, R. L. P., Vieira, R. S., Nicolazzi, L., Martins, D. (2016). Book chapter. Graph-Based Modelling in Engineering. Ed. Zawíslak, S and Rysínski, J. Chapter 9: Three-Dimensional Analysis of Vehicle Stability Using Graph Theory. Springer International Publishing, Switzerland. ISBN 978-3319390185.

DOI 10:1007=978-3-319-39020-8_9.

Muñoz, A.; Carreño, Y.; Pardo, A. (2012). Intelligent Control Systems for Vehicular Traffic. Revista Colombiana de Tecnologías de Avanzada. ISSN: 1692-7257 - v. 2, - n. 20, p 9-16.

Rill, G. (2011). Road Vehicle Dynamics: Fundamentals and Modeling. Boca Ratón, Florida: CRC Press. ISBN 978-1-4398-38983.

Torres, C.; Archila, J.; Tronco, M.; Becker, M.; Viera, A.; Tiberti, A. (2013). Kinematic study of an agricultural robotic platform. Revista Colombiana de Tecnologias de Avanzada. ISSN: 1692-7257 - v. 2, - n. 22, p 131-137.
Winkler, C. (1987). Experimental Determination of the Rollover Threshold of Four TractorSemitrailer Combination Vehicles. UMTRI Research Review, The University of Michigan Transportation Research Institute. Ann Arbor, Detroit.

Winkler, C. (2000). Rollover of Heavy Commercial Vehicles. UMTRI Research Review, The University of Michigan Transportation Research Institute, v. 31, n. 4, p. 1-20. 